\title{
Drop-on-Demand Printing as Novel Method of Oil Supply in Elastohydrodynamic Lubrication
}

\author{
W. M. van der Kruk ${ }^{1}$-S. A. Smit ${ }^{1} \cdot$ T. J. Segers ${ }^{2} \cdot$ X. M. Li ${ }^{3} \cdot$ C. H. Venner ${ }^{1}$ (I)
}

Received: 4 March 2019 / Accepted: 13 July 2019 / Published online: 22 July 2019

(c) The Author(s) 2019

\begin{abstract}
The feasibility of drop-on-demand printing as a novel method for oil supply in elastohydrodynamic lubrication was tested for a single ball-on-disk contact to allow precise lubrication control at a minimal level of lubricant. It is shown that it is feasible to jet droplets of $3.3 \mathrm{pL}$ for an oil of $46 \mathrm{mPa}$ s viscosity at a controlled frequency which, for the conditions considered, results in droplets on the surface with a radius significantly smaller than the Hertzian contact radius. Experimental results of the film thickness evolution over time are presented from dry contact to fully flooded contact with different supply frequencies and a total oil usage of approximately $40 \mathrm{~nL}$. By means of numerical simulation, the details of droplet ingestion into the contact are illustrated. It is shown that the central film thickness over time can accurately be predicted. The theoretical and experimental results provide a very strong incentive for further investigation of drop-on-demand printing as method to achieve optimal (re)lubrication at minimum level in the realistic setting of a rolling bearing.
\end{abstract}

Keywords Elastohydrodynamic lubrication $\cdot$ Replenishment $\cdot$ Drop-on-demand $\cdot$ Starvation

\section{List of Symbols}

\section{Lubricated Contact Variables and Parameters}

$a \quad$ Hertzian contact radius $a=\left((3 F R) /\left(E^{\prime}\right)\right)^{1 / 3}(\mathrm{~m})$

$c \quad$ Hertzian approach $c=a^{2} /(2 R)(\mathrm{m})$

$E \quad$ Modulus of elasticity (Pa)

$E^{\prime} \quad$ Reduced modulus of elasticity

$2 / E^{\prime}=\left(1-v_{1}^{2}\right) / E_{1}+\left(1-v_{2}^{2}\right) / E_{2}(\mathrm{~Pa})$

$F \quad$ Contact load (N)

$G \quad$ Dimensionless material parameter $G=\alpha E^{\prime}(-)$

$h \quad$ Film thickness (m)

$h_{c} \quad$ Film thickness at center of contact (m)

Electronic supplementary material The online version of this article (https://doi.org/10.1007/s11249-019-1208-1) contains supplementary material, which is available to authorized users.

\section{H. Venner}

c.h.venner@utwente.nl

1 Faculty of Engineering Technology, University of Twente, P.O. Box 217, 7500, AE, Enschede, The Netherlands

2 Faculty of Science and Technology, University of Twente, P.O. Box 217, 7500, AE, Enschede, The Netherlands

3 School of Mechanical Engineering, Qingdao University of Technology, No. 777 Jialingjiang Road, Huangdao District, Qingdao 266520, China $h_{\text {cff }} \quad$ Film thickness at center of contact fully flooded (m)

$H \quad$ Dimensionless film thickness $H=h / c(-)$

$H_{c} \quad$ Dimensionless film thickness center of contact

$H_{\text {cff }} \quad$ Dimensionless central film thickness fully flooded contact $(-)$

$h_{\text {oil }} \quad$ Oil layer thickness (m)

$H_{\text {oil }} \quad$ Dimensionless oil layer thickness $H_{\text {oil }}=h_{\text {oil }} / c$ $(-)$

$l_{t} \quad$ Track length $l_{t}=2 \pi\left(R_{x}+R_{\text {disk }}\right)(\mathrm{m})$

$L \quad$ Moes dimensionless lubricant parameter $L=G(2 U)^{1 / 4}(-)$

$M \quad$ Moes dimensionless load parameter $M=W(2 U)^{-3 / 4}(-)$

Pressure $(\mathrm{Pa})$

$p_{h} \quad$ Maximum Hertzian dry contact pressure $p_{h}=(3 F) /\left(2 \pi a^{2}\right)(\mathrm{Pa})$

$P \quad$ Dimensionless pressure $P=p / p_{h}(-)$

$r_{c} \quad$ Ratio of oil layer thickness to central film fully flooded $r_{c}=H_{\text {oil }} /\left(\bar{\rho}_{c} H_{\text {cff }}\right)(-)$

$R \quad$ Reduced radius of curvature $1 / R=1 / R_{x}+1 / R_{y}$ (m)

$R_{x} \quad$ Radius of curvature in $x$ dimension $1 / R_{x}=1 / R_{x 1}+1 / R_{x 2}(\mathrm{~m})$

$R_{y} \quad$ Radius of curvature in $y$ dimension $1 / R_{y}=1 / R_{y 1}+1 / R_{y 2}(\mathrm{~m})$ 


$\begin{array}{llll}R_{x 1}, R_{x 2} & \text { Radius of curvature surface } 1,2 \text { in } x(\mathrm{~m}) & f & \text { Droplet ejection frequency }\left(\mathrm{s}^{-1}\right) \\ R_{y 1}, R_{y 2} & \text { Radius of curvature surface } 1,2 \text { in } y(\mathrm{~m}) & F_{d} & \text { Dimensionless droplet frequency } F_{d}=f \frac{a}{u_{m}}(-) \\ R_{\text {disk }} & \begin{array}{l}\text { Radius of rolling track on disk in experiment } \\ \text { (m) }\end{array} & \operatorname{Re} & \text { Reynolds number } \operatorname{Re}=\frac{\text { oud }}{\eta}(-) \\ \mathcal{R}_{c} & \text { Ratio central film to fully flooded value } & u_{n} & \text { Droplet ejection velocity at the nozzle }\left(\mathrm{ms}^{-1}\right) \\ & \mathcal{R}_{c}=H_{c} / H_{\mathrm{cff}}(-) & V_{d} & \text { Ejected droplet volume }\left(\mathrm{m}^{3}\right) \\ t & \text { Time }(\mathrm{s}) & V_{\mathrm{ca}} & \text { Spherical cap volume on surface }\left(\mathrm{m}^{3}\right) \\ T & \text { Dimensionless time } T=t u_{m} / a(-) & \text { We } & \text { Weber number We }=\frac{\rho u^{2} d}{\sigma}(-) \\ u & \text { Surface velocity }\left(\mathrm{ms}^{-1}\right) & Z_{0} & \text { Parameter in dimensionless droplet shape on } \\ u_{m} & \text { Average (entrainment) velocity } u_{m}=\left(u_{1}+u_{2}\right) / 2 & \varepsilon & \text { surface: } Z_{0}=r_{\mathrm{ca}} \cos \left(\theta_{c}\right) / c(-) \\ & \left(\mathrm{ms}^{-1}\right) & \theta_{c} & \text { Efficiency of a droplet ingestion }(-) \\ U & \text { Dimensionless speed parameter } & \sigma & \text { Contact angle }(-)\end{array}$

$W \quad$ Dimensionless load parameter $W=F /\left(E^{\prime} R_{x}^{2}\right)$ (-)

$x, x^{\prime} \quad$ Coordinates in direction of rolling $(\mathrm{m})$

$X, X^{\prime} \quad$ Dimensionless coordinates in direction of rolling $X=x / a, X^{\prime}=x^{\prime} / a(\mathrm{~m})$

$y, y^{\prime} \quad$ Coordinates perpendicular to direction of rolling (m)

$Y, Y^{\prime} \quad$ Dimensionless coordinates perpendicular to direction of rolling $(-)$

$\alpha \quad$ Pressure viscosity index oil $\left(\mathrm{Pa}^{-1}\right)$

$\delta \quad$ Mutual separation (m)

$\delta_{t} \quad$ Time delay (s)

$\Delta \quad$ Dimensionless mutual separation $\Delta=\delta / c(-)$

$\Delta_{X} \quad$ Mesh size grid dimensionless $X$ direction (-)

$\Delta_{Y} \quad$ Mesh size grid dimensionless $Y$ direction (-)

$\Delta_{T} \quad$ Timestep dimensionless time (-)

$\rho \quad$ Lubricant density $\left(\mathrm{kg} \mathrm{m}^{-3}\right)$

$\rho_{0} \quad$ Lubricant density at ambient pressure $\left(\mathrm{kg} \mathrm{m}^{-3}\right)$

$\bar{\rho} \quad$ Lubricant dimensionless density $\bar{\rho}=\rho / \rho_{0}(-)$

$\eta \quad$ Lubricant viscosity (Pa s)

$\eta_{0} \quad$ Lubricant viscosity at ambient pressure (Pa s)

$\eta_{a} \quad$ Viscosity of air (Pa s)

$\bar{\eta} \quad$ Dimensionless viscosity $\bar{\eta}=\eta / \eta_{0}(-)$

$\theta \quad$ Fractional film content $\theta=h_{\mathrm{oil}} / h(-)$

$v \quad$ Poisson's ratio $(-)$

$\lambda$ Dimensionless parameter in Reynolds equation $\lambda=\left(12 u_{m} \eta_{0} a\right) /\left(c^{2} p_{h}\right)(-)$

$\gamma \quad$ Side flow parameter in starved film reduction equation $(-)$

\section{Droplet Variables}

Bo $\quad$ Bond number Bo $=\left(\rho_{0}-\rho_{a}\right) d_{d}^{2} g / \sigma(-)$

$d_{n} \quad$ Diameter of the nozzle $(\mathrm{m})$

$D_{p} \quad$ Dimensionless height of precursor layer of oil (-)

$d_{d} \quad$ Diameter of ejected droplet (m)

$d_{\text {ca }} \quad$ Spherical cap droplet shape on surface (m)

$D_{p} \quad$ Dimensionless height of precursor layer of oil (-)

$D_{\text {ca }} \quad$ Dimensionless droplet shape $D_{\text {ca }}=d_{\text {ca }} / c(-)$

\section{Introduction}

To mitigate the possible consequences of climate change, an unprecedented effort is required to change energy generation, energy usage, and all related technological processes toward a long-term sustainable development. This awareness has already led to restrictive bounds set on product development, and is affecting all areas of technology. Current technologies are pushed to their limits by increasingly strict efficiency demands, minimization of material use, and environmental regulations, while new sustainable applications and energy technologies are rapidly developing. A major fraction of the global energy usage originates from tribological contacts to overcome friction and to remanufacture worn parts [1]. Improved control of friction at a lower level by optimizing lubrication in machine components such as bearings and gears is a potential energy saver. This is a major challenge since the conditions at which lubricated contacts have to operate reliably are becoming more severe. Higher temperatures and higher loads lead to thinner lubricant films with increasing sensitivity to effects of surface roughness, dynamic loading and pressure disturbances resulting from subsurface material heterogeneity. Lubricant composition changes, as e.g., particular additives which may end up in emissions have to be replaced, as well as there is a drive for more green lubricants. Finally, in many cases supply is significantly reduced to minimize frictional losses from churning or sloshing, or as a result of operating conditions where minimal reflow is achieved due to e.g., high speed, relatively high viscosity, or large size. Lubricated contacts have to operate more reliably under conditions at which the fully flooded film thickness limit, predicted from formulas such as presented by Hamrock and Dowson [2] and Nijenbanning [3], is not achieved and the contacts operate in the "starved regime" [4]. From a film thickness point of view, starved contacts are by definition very efficient. In fully flooded EHL contacts, over $90 \%$ of the lubricant 
is removed by sideflow and not ingested into the contact to separate the surfaces, whereas in significantly starved contacts, almost the entire lubricant on the track ends up in the contact. However, the risk of insufficient lubrication is often large in terms of potential hazardous consequences of failure, damage, and downtime. The amount of lubricant supplied, either in relubrication intervals or by oil-air or oil-mist lubrication, is generally much larger than what is actually needed by the contacts simply to ensure that the probability of sufficient oil reaching the contact, e.g., by surface coverage, is high enough.

Booser and Wilcock [5] have already demonstrated that providing only $1 \mathrm{mg}$ of oil was sufficient to run a standard bearing for a considerable number of hours, and thus the amount of oil needed to provide sufficient surface coverage for lubrication and friction minimization to be very small. Even after taking into account that in many applications, the lubricant may fulfill a role for cooling purposes, it still appears that a significant reduction in lubricant use can be achieved by lubrication systems which act on the same scale as the contact volume. Realizing such a system and demonstrating its feasibility for lubricating a single EHL contact is the topic of the present study.

Starvation redirects the focus from the film thickness inside the contact to the relation between the supply to the contact and its relation to the film formed inside the contact. Studies of starved lubrication date back to Wedeven [4]. Pemberton and Cameron [6] were the first to study the flow around the contact showing the characteristic butterflyshaped oil reservoir. Chevalier et al. and Damiens et al. [7-11] studied starved contacts under steady-state conditions and found a simple predictive relation based on a single parameter which only depends on the inlet length. Starved contact results for time-varying contacts were presented in [12-14]. Using insights obtained from the starved lubrication studies, van Zoelen [15-17] introduced a new approach based on free surface layer modeling of contacts. He showed that in the absence of resupply, the film thickness rapidly decays in a period of only minutes, and provided experimental validation for a single contact. Extending the model to bearing models, a sufficient film thickness level was predicted to exist only during a number of overrollings many orders of magnitude lower than the observed lubrication life of bearings. This indicates that resupply/redistribution mechanisms play a crucial role in bearings, i.e., centrifugal effects, scraping effects, or cage redistribution. However, these mechanisms are "passive" in the sense that they act only when lubricant is available in abundance, like grease bleeding oil on the side of the track or grease located at/ near the cage.

In this paper, we investigate the possibility to achieve a significant further reduction of lubricant supply by inkjet technology. In inkjet printing, liquid is expelled through a nozzle to form droplets that land on a substrate [18]. One of the techniques used is drop-on-demand printing that either uses a piezo-acoustic or thermal actuator to generate a droplet [19, 20]. In the last decade, the understanding of the droplet formation process has increased by research on the fluid motion inside the nozzle [21] and the inflight dynamics of micrometersized droplets [22]. Inkjet printing has become more reliable through understanding of air bubble entrapment and dirt particle dynamics such that picoliter droplets can now be reproducibly jetted [23].

The necessary volume of lubricant required in an EHL contact, and the droplet volume of inkjets are of the same order. Therefore, the usage of inkjet printed droplets is an interesting topic for highly controlled minimal supply lubrication. Li [24] initiated research on the behavior of droplets passing through an EHL contact from the viewpoint of oil-air lubrication. Various aspects of the effect of the entrainment speed, viscosity, and wettability have been observed both experimentally [25, 26] and numerically [27]. Here, we advance to significantly smaller droplets and control of the lubrication with a drop-ondemand printing technology. One of the main challenges is that the viscosity of lubricating oils is often much larger than the viscosity of inkjet fluids, so that it is more difficult to create small droplets. In an earlier work [28], a T9 oil of viscosity $15.7 \mathrm{mPa}$ s was used successfully for generating droplets of $195 \mathrm{pL}$ with a radius of $400 \mu \mathrm{m}$. In this paper, we show that by means of a heated printing nozzle, it is possible to derive much smaller volume droplets for a higher viscous oil. It is demonstrated that, with optical interferometry film thickness measurements, a build-up of a lubricant film from dry to fully flooded state can be realized using a drop-on-demand system that ejects oil droplets of only $3.3 \mathrm{pL}$. This is done in a controlled manner with an amount of lubricant of the order of nanoliters. From numerical simulations, it is shown that in the severely starved regime, $85 \%$ of the volume of each drop is actually ingested into the contact. Based on this result, a simple model is presented that accurately predicts the lubrication build-up as observed in the experiments for different droplet supply frequencies. The successful single contact results presented in this paper demonstrate that drop-on-demand printing is a very interesting technique to further investigate, e.g., in an experimental setup with an actual rolling bearing.

\section{Theory}

The Reynolds equation with dimensionless variables, see Nomenclature, for unidirectional entrainment taking into account time-varying conditions is given by 


$$
\begin{aligned}
\frac{\partial}{\partial X}\left(\frac{\rho \bar{H}^{3}}{\bar{\eta} \lambda} \frac{\partial P}{\partial X}\right)+\frac{\partial}{\partial Y}\left(\frac{\rho \bar{H}^{3}}{\bar{\eta} \lambda} \frac{\partial P}{\partial Y}\right), \\
\quad=\frac{\partial(\bar{\rho} \theta H)}{\partial X}+\frac{\partial(\bar{\rho} \theta H)}{\partial T} .
\end{aligned}
$$

where $X$ and $Y$ are the coordinates in the plane of the lubricant film with $X$ in the direction of entrainment. A rectangular domain $X_{a} \leq X \leq X_{b} \wedge-Y_{a} \leq Y \leq Y_{b}$ is used. $P$ and $H$ denote the dimensionless pressure and film thickness. $\theta$ is the fractional film content, introduced by Elrod [29] to model starved lubrication, defined as $\theta=H_{\text {oil }} / H$, the ratio of the height of the lubricant layer on the surfaces $H_{\text {oil }}$ and the actual gap height. The solution is subject to the complementarity condition:

$\left\{\begin{array}{lll}0 \leq \theta<1 & \& & P=0 \\ \theta=1 & \& & P>0\end{array}\right.$

Under these conditions, Eq. (1) becomes an equation for $P$ in regions where $\theta=1$ and an equation for $\theta$ when $P=0$. The dimensionless density and viscosity are taken from the empirical Dowson-Higginson [30] and Roelands [31] equations, respectively. The dimensionless film thickness equation is given by

$$
\begin{aligned}
H(X, Y, T)= & -\Delta(T)+\frac{1}{2}\left(X^{2}+Y^{2}\right) \\
& +\frac{2}{\pi^{2}} \iint_{A} \frac{P(X, Y, T)}{\sqrt{\left(X-X^{\prime}\right)^{2}+\left(Y-Y^{\prime}\right)^{2}}} \mathrm{~d} X^{\prime} \mathrm{d} Y^{\prime},
\end{aligned}
$$

where the integral represents the elastic deformation of the surfaces. The dimensionless mutual approach $\Delta(T)$ is determined by the dynamic behavior of the contact. Neglecting acceleration terms this leads to the force balance equation:

$\iint_{A} P(X, Y, T) \mathrm{d} X \mathrm{~d} Y=\frac{2 \pi}{3} \quad \forall T$.

The solution is subject to the boundary condition $P=0$ on all sides of the domain. At the inlet side $H_{\text {oil }}=H_{\text {oil }}(Y, T)$ is given as the (equivalent) thickness of a layer of lubricant moving with the surface. When $H_{\text {oil }}$ is so large that, at the inlet of the domain, $\theta=H_{\text {oil }} / H=1$ ensues over the entire width and in time, then obviously the result will be the fully flooded solution. Let $H_{\text {cff }}$ denote the value of the film thickness in the center of the contact for this case. For smaller values of $H_{\text {oil }}$, one obtains a starved solution with the inlet meniscus a function of $(X, Y)$ depending on the degree of starvation and the profile $H_{\mathrm{oil}}\left(X_{a}, Y\right)$. Let the central film thickness for this case be denoted by $H_{c}$.

Chevalier [7, 8] investigated the dependence of the film thickness in the center on the degree of starvation, in a circular contact by monitoring the central film thickness reduction $\mathcal{R}_{c}=H_{c} / H_{\text {cff }}$ as a function of the (uniform) oil layer thickness $H_{\text {oil }}$ in the inlet. Defining

$r_{c}=\frac{H_{\mathrm{oil}}}{\overline{\rho_{c}} H_{\mathrm{cff}}}$,

where $\bar{\rho}$ is the dimensionless density at the center of the contact, approximated as $\bar{\rho}_{c}=\bar{\rho}\left(p_{h}\right)$, they derived the following relation to hold with good accuracy:

$\mathcal{R}_{c}=\frac{H_{c}}{H_{\mathrm{cff}}} \approx \frac{r_{c}}{\sqrt[\gamma]{1+r_{c}^{\gamma}}}$

The relation between $r_{c}$ and $\mathcal{R}_{c}$ is fully characterized by the parameter $\gamma$, which was determined from the numerical results obtained for a given contact operating at specific conditions at varying degrees of starvation $r_{c}$, i.e., with varying values of $H_{\mathrm{oil}}$. For $H_{\mathrm{oil}} \ll H_{\mathrm{cff}}$, one finds $H_{c} \approx H_{\mathrm{oil}} / \bar{\rho}_{c}$ indicating that no side flow occurs, and the entire lubricant is used for film formation. When $H_{\text {oil }} \gg H_{\text {cff }}$, the central film thickness will approximate the value for the fully flooded contact, see also Sect. 4.2. $\gamma$ represents the resistance to side flow and depends on the operating conditions. In Damiens [10], the work of Chevalier is extended to wide elliptic contacts, and it is shown that $\gamma$ is determined by the inlet length of the contact, which can be written as $\sqrt{M / L}$ for circular contacts with $L$ and $M$, the Moes dimensionless parameters. In $[7,10]$, it is also shown how $\gamma$ can be determined from single contact ball-on-disk experiments.

In this study, Eq. (6) will be used to develop a simple model that predicts the film thickness evolution as a function of time using a drop-on-demand system, which jets droplets with a particular frequency and shape. To determine how much of the volume of a single droplet on the surface is ingested into the contact, a full numerical solution of the time-dependent starved problem is done with a droplet $D(X, Y, T)$ deposited on a thin precursor layer $D_{p}$ passing through the contact:

$H_{\text {oil }}(X, Y, T)=D_{p}+D_{\text {ca }}(X, Y, T)$

This precursor layer in fact implies that the dry region around the droplet is modeled as a heavily starved contact. For droplets of a sufficiently large height relative to the film thickness, the error that is made compared to the real case, in which the surface is dry, will be small. The advantage is that the entire numerical simulation can be done with the starved Reynolds equation. $D_{\text {ca }}$ is determined from the spherical cap formed by an ejected droplet deposited on the surface moving with the rolling velocity starting at position $X_{0}$ in the experimental study.

Let the droplet generated by the nozzle have volume $V_{d}=4 \pi\left(d_{d} / 2\right)^{3} / 3$. After stabilization following the impact on the disk, it forms a spherical cap with radius $r_{\text {ca }}$ and 
wetting angle $\theta_{c}$. Assuming the location to be central on the track $y=0$ and at position $x=x_{\mathrm{ca}}$, its form as a function of the surface coordinates $x$ and $y$ is given by

$d_{\mathrm{ca}}(x, y)=-r_{\mathrm{ca}} \cos \left(\theta_{c}\right)+\left(r_{\mathrm{ca}}^{2}-\left(x-x_{\mathrm{ca}}\right)^{2}-y^{2}\right)$

for $\left(x-x_{\mathrm{ca}}\right)^{2}+y^{2} \leq a_{\mathrm{ca}}^{2}$, with $a_{\mathrm{ca}}$ the radius on the surface, and $d_{\mathrm{ca}}=0$ otherwise. By definition $a_{\mathrm{ca}}=r_{\mathrm{ca}} \sin \left(\theta_{c}\right)$, and the height at the center location $d_{\mathrm{ca}}(0,0)=r_{\mathrm{ca}}\left(1-\cos \left(\theta_{c}\right)\right)$. The volume of the cap is given by

$V_{\mathrm{ca}}=\frac{\pi}{6} d_{\mathrm{ca}}(0,0)\left(3 r_{\mathrm{ca}}^{2} \sin ^{2}\left(\theta_{c}\right)+d_{\mathrm{ca}}(0,0)^{2}\right)$

Assuming no fluid is lost during jetting and impact on the disk $V_{\text {ca }}=V_{d}$ and the wetting angle $\theta_{c}$ known from a measurement $r_{\mathrm{ca}}$ can be solved from Eq. (9). Scaling the surface coordinates $x$ and $y$ on the Hertzian contact radius and the droplet height on the maximum Hertzian deformation $c=a^{2} /(2 R)$ consistent with the film thickness scaling and oil layer scaling, one obtains the dimensionless shape of the droplet on the disk:

$D_{\mathrm{ca}}(X, Y)=-Z_{0}+\mathcal{Z} \sqrt{\frac{r_{\mathrm{ca}}^{2}}{a^{2}}-\left(X-X_{\mathrm{ca}}\right)^{2}-Y^{2}}$.

with $Z_{0}=r_{\mathrm{ca}} \cos \left(\theta_{c}\right) / c$ Finally, assuming the droplet starts at position $X_{0}$ and moves toward the contact with the speed of the disk under pure rolling conditions:

$X_{\mathrm{ca}}=X_{0}+T$

giving

$D_{\text {ca }}(X, Y, T)=-Z_{0}+\mathcal{Z} \sqrt{\left.\frac{r_{\mathrm{ca}}^{2}}{a^{2}}-\left(X-X_{0}-T\right)\right)^{2}-Y^{2}}$.

For the precursor layer, a fixed height $D_{p}$ will be taken. The actual values used in the simulations are given in Sect. 4.3.

\section{Methods}

\subsection{Numerical Method}

The Eqs. (1)-(4) were discretized using a finite difference discretization on a uniform grid with mesh size $\Delta_{X}=\Delta_{Y}$ and timestep $\Delta_{T}$ covering the domain $-2.5 \leq X \leq 1.5 \wedge-2 \leq Y \leq 2$. The discretization is second order in space and time. A second central scheme was used for the pressure flow terms of Eq. (1) and a narrow upstream scheme, $N U 2$, for the wedge and squeeze term, see [32,33]. The $N U 2$ scheme has the advantage of a zero leading order truncation error for the characteristic direction $X=T$ when choosing the dimensionless timestep $\Delta_{T}=\Delta_{X}$. It provides more accurate solutions on coarser grids than separate standard upstream discretization of the wedge and squeeze term, as a result of the significantly lower artificial diffusivity. The discrete equations at each timestep were solved using Multigrid techniques with Multilevel Multi-Integration for the fast evaluation of the multi-summation representing the elastic deformation, see [14, 33, 34]. For each timestep, the discrete equations are solved to an error that is small compared to the discretization error using a few multigrid $W$-cycles. The calculations were carried out using the finest grid of $513 \times$ 513 points.

\subsection{Experimental Method}

Optical interferometry was used to perform film thickness measurements. This technique dates back to the work of Westlake and Cameron [35] and Foord [36]. In the early works, central film thickness measurements were carried out by directing light, obtained from a small section of the width of the contact, to a spectrometer for the analysis of the maximum wavelength of maximum interference. A silica spacer layer was introduced by Johnston [37] to make thin film measurements possible. Subsequently, the Spacer Layer Imaging Method was introduced by Cann [38] to obtain full maps of the entire contact. The experimental setup used here (Fig. 1) is a modified version of the EHD2 Ultra Thin Film Measurement System manufactured by PCS Instruments. It was used for both measurements (ULTRA and SLIM) with a resolution of $\pm 5 \mathrm{~nm}$. The modification involves the replacement of the

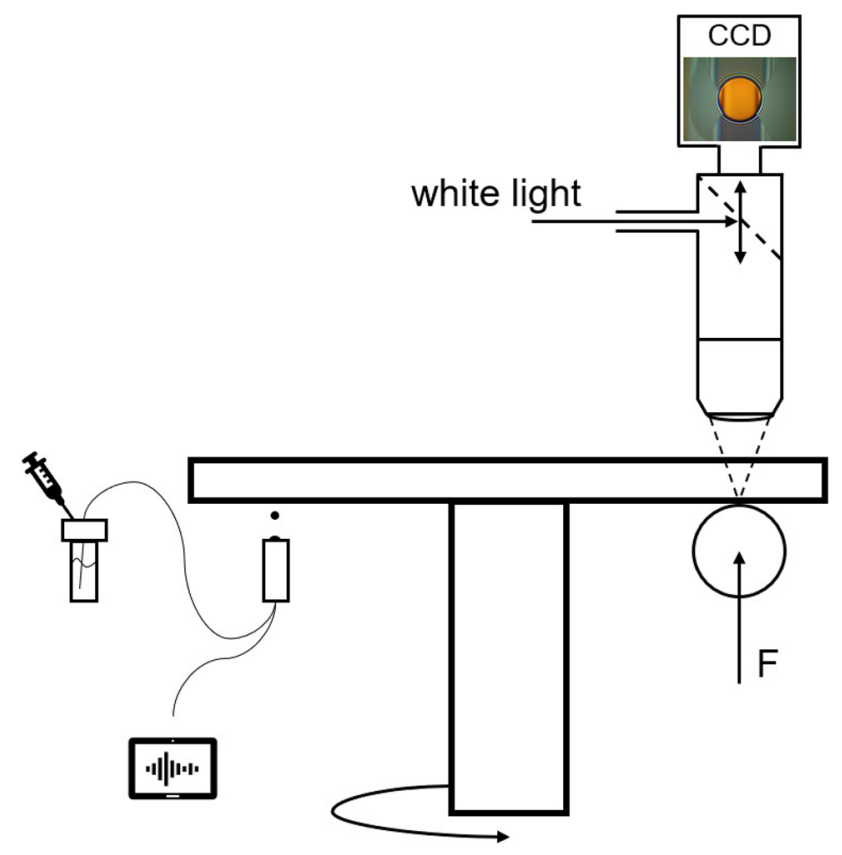

Fig. 1 Schematic overview of the ball-on-disk setup that is lubricated by drop-on-demand inkjet printing 
standard lubricant reservoir to allow open access to the disk for the nozzle of the drop-on-demand system.

The setup consists of a steel ball and a glass disk that is coated on the top side with $\mathrm{MgF}_{2}$ and on the bottom side with a semireflective chromium layer of $20 \mathrm{~nm}$ as well as a silica layer of $130 \mathrm{~nm}$ for the SLIM measurements and about $500 \mathrm{~nm}$ for the ULTRA measurements. The contact was lubricated with Shell High Viscosity Index 60 (HVI-60) oil. Properties of the solids and lubricant as well as nominal test parameters and the associated values of the Moes dimensionless numbers $M$ and $L$ can be found in Table 1. A CCD camera (JAI Apex CV-M9CL) equipped with a magnifying objective (Zeiss $5 \times$ ) was used to capture images of the contact. The spatial resolution was $0.93 \times 0.93 \mu^{2}$ over an area of $952 \times 714 \mu \mathrm{m}^{2}$. For the SLIM, images the RGB values of each pixel were matched to calibration data to obtain a film thickness map of the full contact.

\subsection{Drop-on-Demand Printing System}

A single piezo-acoustic drop-on-demand (DOD) printhead with a $70 \mu \mathrm{m}$ diameter nozzle (Microdrop MD-K-140) was used to generate a single droplet upon each piezo driving pulse [19]. In DOD inkjet printing, typically, for a standard square wave piezo driving pulse, a droplet is generated with a diameter similar to the nozzle exit. However, using more complex piezo driving waveforms, the droplet diameter can be decreased [39, 40]. Here, a two-step piezo driving waveform was used, see Fig. 2. The piezo driving waveform was produced by an arbitrary waveform generator (Iso-Tech AFG-21555) and amplified by 50 times (Falco Systems WMA-300). The amplitudes of the plateaus $\left(V_{1}\right.$ and $\left.V_{2}\right)$ and the lengths of the first- and second plateaus $\left(t_{1}\right.$ and $\left.t_{2}\right)$ were varied to minimize the droplet volume. The droplet volume was optically measured using a microscope (Olympus

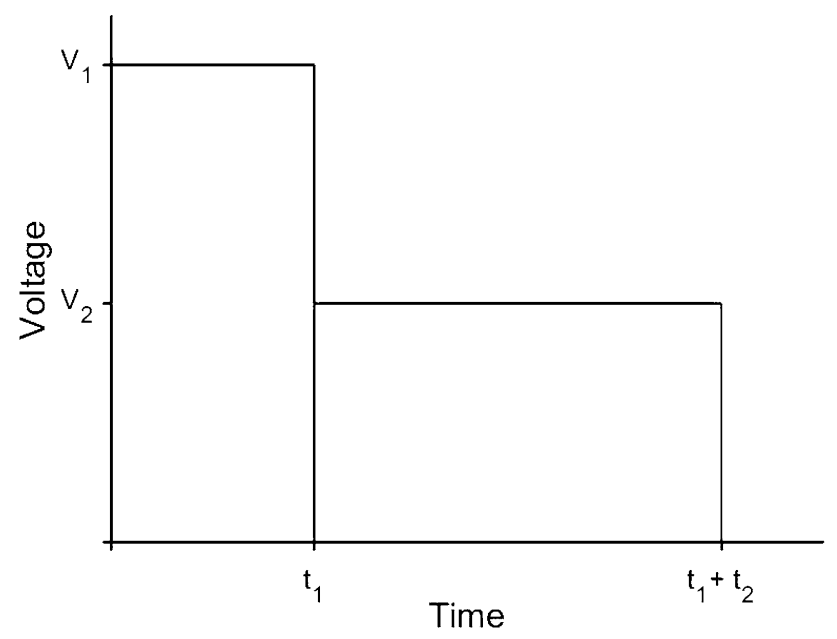

Fig. 2 Square wave signal that is used for actuation
BX-FM modular system) equipped with a 10 times magnifying objective (Olympus SLMPLN) and a CCD camera (Lumenera LW135M). To minimize motion blur, illumination was provided by laser-induced fluorescence [41], i.e., a $7 \mathrm{~ns}$ laser pulse (Nd:YAG, $532 \mathrm{~nm}$ ) was coupled into a fluorescent diffuser (LaVision) to provide a noncoherent $8 \mathrm{~ns}$ light flash. The delay of the light flash with respect to the start of the piezo-driving waveform was varied with nanosecond precision using a pulse-delay generator (Berkeley Nucleonics, BNC 575) in order to stroboscopically visualize the full droplet formation process. Before the experiments, the oil meniscus in the printhead was positioned at the nozzle exit $\left(d_{n}=70 \mu \mathrm{m}\right)$ through pressure control on the oil reservoir.

In inkjet printing, the conditions under which droplets can be successfully jetted can be quantified in terms of the Reynolds- and the Weber number, i.e., $W e^{1 / 2} R e^{1 / 4}<50$, $0.1 \leq W e \leq 1$, and $W e>4$ [42-44]. Therefore, to be able to jet high-viscosity oils, the tip of the nozzle was heated to $90{ }^{\circ} \mathrm{C}$ to decrease the oil viscosity from $46.3 \mathrm{mPa}$ s at $20^{\circ} \mathrm{C}$ to $4.4 \mathrm{mPa}$ s, see Table 1 .

\section{Results}

\subsection{Droplet Generation}

The piezo driving waveform at which the droplet volume was at minimum was found to have the following parameters: $V_{1}=128 \mathrm{~V}, V_{2}=64 \mathrm{~V}, t_{1}=16 \mu \mathrm{s}$, and $t_{2}=32 \mu \mathrm{s}$ (Fig. 2). For these parameters, the full jet formation process is show in Fig. 3 for upward and downward jetting. Since the Bond number $B o \ll 1$, gravitational effects can be neglected. Indeed, Fig. 3 shows highly similar results for jetting in the direction of gravity and for jetting in the direction against gravity. The minor differences result most likely from a slightly different meniscus position and/or different hydrodynamic pressure in the oil reservoir. Thus, drop-ondemand printing is well suited to deposit droplets from any angle at locations that are hard to reach. The images have been analyzed to extract the droplet diameters, volumes, and velocities, see Table 2. The upward jetted droplet had a diameter of $d_{d}=18.6 \mu \mathrm{m}\left(V_{d}=3.3 \mathrm{pL}\right)$ and a velocity of

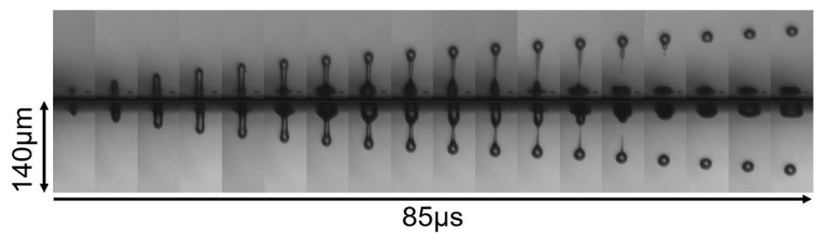

Fig. 3 Image sequence of the jetting process with the corresponding length and time scale. The top image shows an upward directed jetting process. The bottom image is in downward direction 
$0.9 \mathrm{~m} \mathrm{~s}^{-1}$. The droplet slows down due to air drag. Based on solving the equation of motion, numerically a distance of $0.02 \mathrm{~m}$ was deemed suitable between the nozzle tip and the rotating disk so that the droplet reaches the disk and is not slowed down too much. From the thermal lumped system analysis of the cooling of the droplet, it can be determined that the time for the oil to cool from 90 to $20{ }^{\circ} \mathrm{C}$ is about $3.3 \mathrm{~ms}$ which ensures that by the time the droplet reaches the contact, it has ambient temperature, so that for lubrication the viscosity at ambient temperature will apply.

\subsection{Fully Flooded and Starved Solution}

For reference, fully flooded central film thickness measurements were done in the velocity range $10-500 \mathrm{~mm} \mathrm{~s}^{-1}$ with

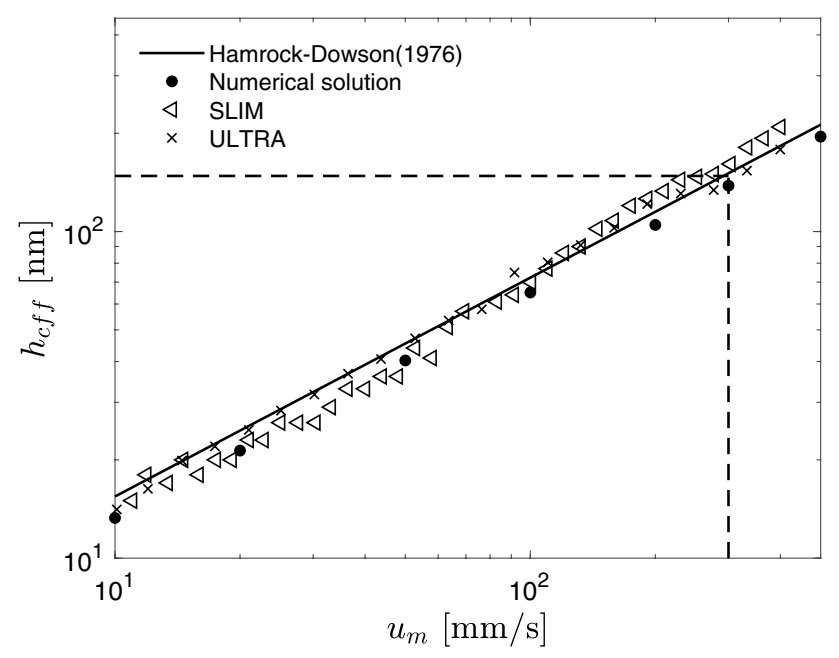

Fig. 4 Central fully flooded film thickness as function of the velocity obtained from the relation of Hamrock-Dowson [2], with the numerical method, the SLIM method, and the ULTRA method. The dotted line indicates the velocity and its corresponding film thickness that will be used in the starved solution the ULTRA and SLIM method. The results for the central film thickness are compared to the results of the full numerical solution and to the prediction of the Hamrock and Dowson [2] relation in Fig. 4. In view of the fact that at room temperature, the viscosity is very sensitive to the temperature, all results agree well. The spectrometry measurements agree with the numerical solution and the Hamrock and Dowson predictions. The SLIM measurements have a slight deviation. The dashed line in the graph indicates the entrainment velocity of $300 \mathrm{~mm} / \mathrm{s}$ according to the operating conditions used in the experiment and in the numerical starved solution.

From numerical solutions, the value of the sideflow parameter $\gamma=3.2$ was determined for $r_{c}=1$ using the Moes' parameters from Table 2. This fully defines Eqs. (5) and (6). Figure 5 presents the prediction together with the numerical solution obtained for seven different values of $H_{\text {oil }}$, which are indicated by markers. The prediction of Damiens fits the numerical data of the steady-state solution well. It shows an approximation of $H_{c}=H_{\text {oil }} / \bar{\rho}_{c}$ for $H_{\text {oil }} \ll H_{\text {cff }}$ and $H_{c}=H_{\text {cff }}$ for $H_{\text {oil }} \gg H_{\text {cff }}$.

\subsection{Droplet Shape on Disk}

The oil droplet forms a spherical cap on the rotating disk following stabilization after impact, as explained in Sect. 2. The contact angle was measured as $\theta_{c}=21.3^{\circ}$, once the droplet is stable from the impact. The wettability in terms of $\theta_{c}$ is an important property of lubrication by droplets, as has been noted by $\mathrm{Li}$ et al. [26]. For a generated droplet volume $V_{d}=3.3 \mathrm{pL}$ from Eq. 9, one obtains a cap radius $r_{\mathrm{ca}}=6.1610^{-5} \mathrm{~m}$, the radius of the droplet on the surface $a_{\text {ca }}=22.4 \mu \mathrm{m}$, and a cap height $d_{\text {ca }}(0,0)=4.2 \mu \mathrm{m}$. Note that this cap height is much bigger than the central film thickness $h_{\mathrm{cff}}=149 \mathrm{~nm}$. However, the radius on the surface $(22.4 \mu \mathrm{m})$ is significantly smaller than the Hertzian contact radius $(2 a=273 \mu \mathrm{m})$.
Fig. 5 Comparison between seven numerical solutions and the prediction of Damiens[11] with $\gamma=3.2$. The other lines show the asymptotes for the limiting cases of $r_{c}$

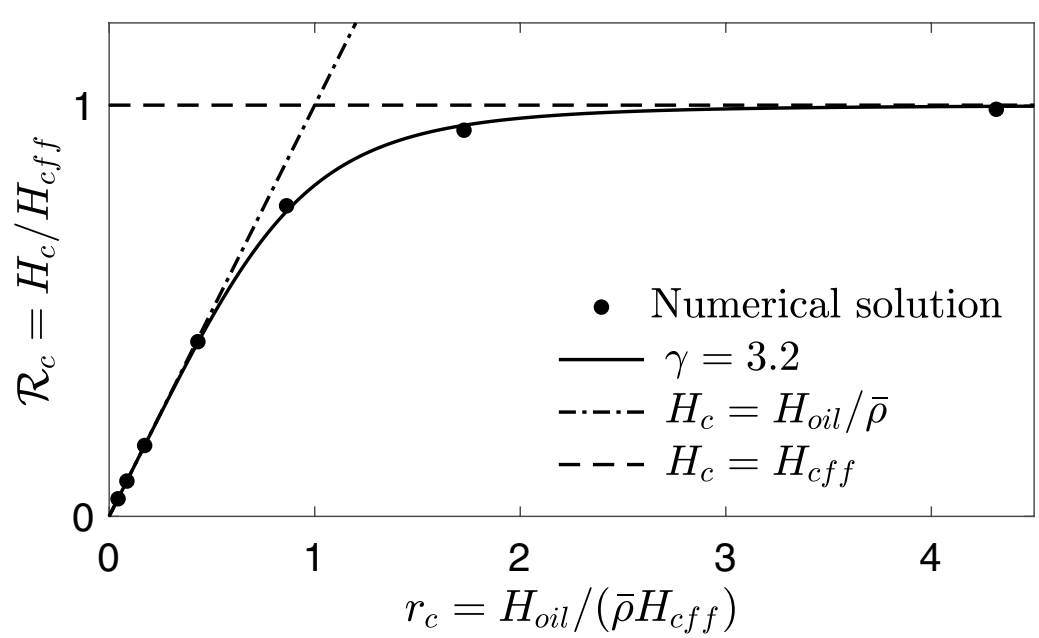


This results in interesting spreading dynamics when the droplet enters the contact. The value of the scale factor of $\mathcal{Z}=2 R / a=70$ is used for the experimental conditions.

\subsection{Single-Droplet Simulation}

The droplet dynamics of a single droplet that is ingested by the contact were modeled using a grid of $513 \times 513$ points. The steady-state starved solution is taken with a precursor layer $\left(D_{p}=0.01\right)$ on the surface. This value was small enough not to affect the results significantly and large enough to ensure a stable numerical solution process throughout the full domain. Subsequently, the evolution over time was simulated for the case of a droplet on top of the precursor layer. The results for a the droplet that is ingested by the contact are presented in Fig. 6 for several
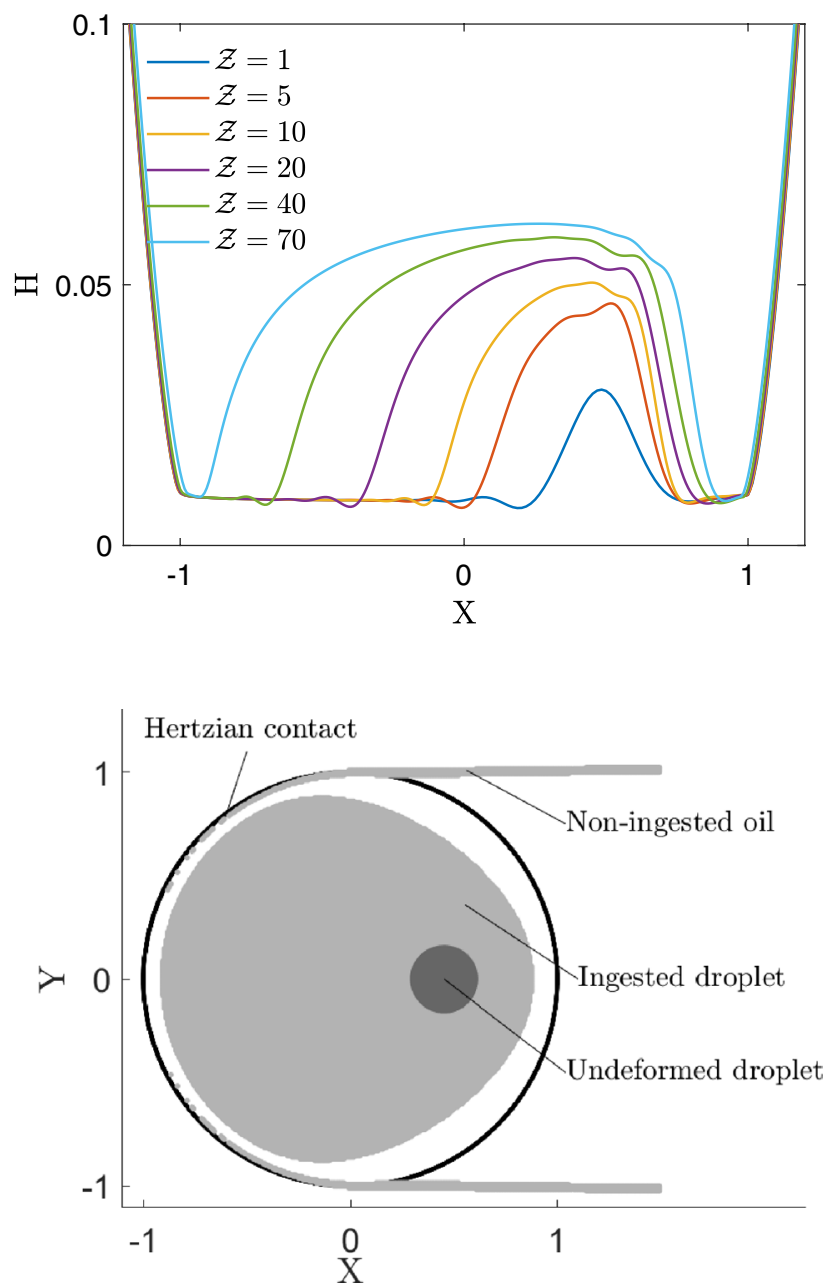

Fig. 6 Top image: numerically obtained film thickness of an ingested droplet using seven different $\mathcal{Z}$-scales. The solution for $\mathcal{Z}=70$ represents the experimentally used droplet. Bottom image: top view of the Hertzian contact, where a droplet is ingested with an efficiency of $\varepsilon=0.85$. The spreading of the droplet in $X$ - and $Y$-direction can be seen compared to the undeformed droplet values of the scale factor $\mathcal{Z}$. This can be interpreted as varying the (dimensionless) droplet height on the disk. When the droplet radius is small relative to the contact, as is the case in our tests, at a small $\mathcal{Z}$, it will create a small film thickness increase moving through the contact. The film thickness modulation is rotationally symmetric. However, with the increasing height, the droplet spreads in the inlet. As a result, it elongates considerably in $X$ and $Y$-directions over the full contact length. It remains symmetrical in the $Y$-direction but becomes asymmetrical relative to the original droplet central position advanced in time along $X$-direction. Figure 6 also shows the region of the contact in which the film thickness is increased due to the ingested droplet, and the original droplet radius. The time variations of the film thickness in pseudo-interferometry graph as well as its profile at both centerlines of the contact for $\mathcal{Z}=70$ are presented in animations in Online Appendix A.1, A.2, and A.3..

From the numerical results, the volume of an ingested amount of lubricant inside the contact (for the experimental condition $\mathcal{Z}=70$ ) was determined to be $2.80 \mathrm{pL}$. This indicates a lubrication efficiency of $\varepsilon=\frac{2.80}{3.3}=0.85$.

Finally, Fig. 7 shows the accuracy of the numerical solution by comparing results obtained at grids with different gridsizes and timesteps. The results show that on the $513 \times$ 513 grid and the associated timestep, the discretization error already is very small.

\subsection{DOD Film Build-Up Model}

By means of the result of the numerical solution of one ingested droplet, the lubricating behavior of multiple

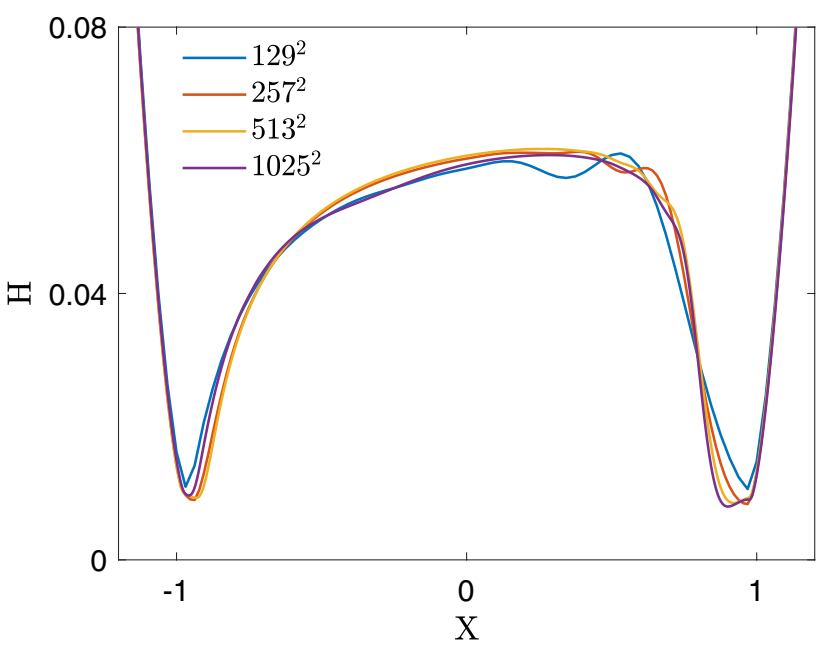

Fig. 7 Numerically obtained film thickness of an ingested droplet using four different finest grid sizes at centerline $X=0$. The figure shows a well converged solution at a $513^{2}$ grid size 


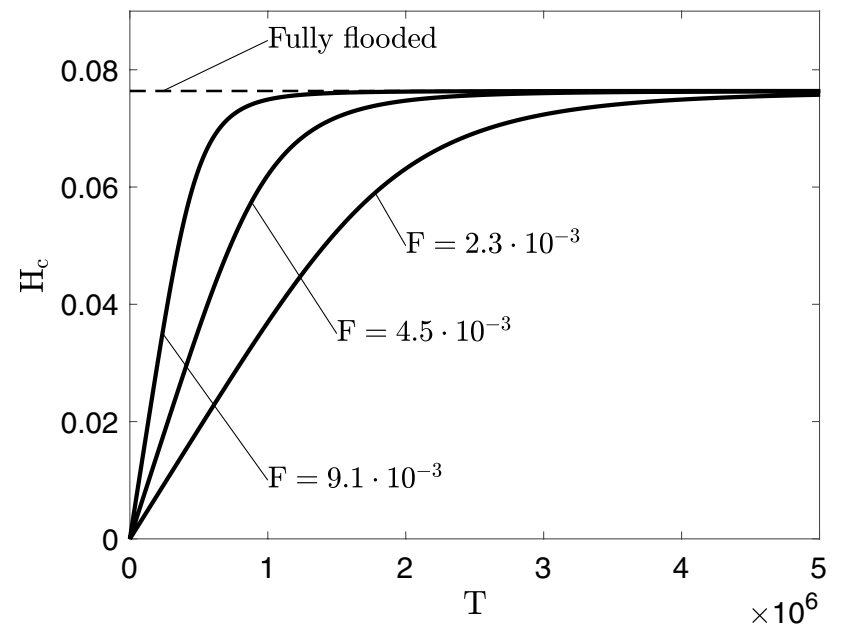

Fig. 8 Central film thickness build-up over time at three different frequencies, according to the presented build-up model

droplets can be predicted, with the assumption that each droplet has the same efficiency $\varepsilon$ and that a droplet is instantly spread over the whole track. In fact, the droplets were printed on the disk at a distance of $u_{m} / f$ from each other. The effective parts of all droplets account for a volume that can be translated to an oil layer thickness $h_{\text {oil }}$ using the width of the contact and the track length:

$V_{\text {oil }}=\varepsilon V_{d} f t=2 a l_{t} h_{\text {oil }}$

or dimensionless

$H_{\text {oil }}=\frac{\varepsilon V_{d}}{2 a c l_{t}} F T$

From this value of the oil layer thickness, the central film thickness can be calculated using Eqs. (5) and (6), which leads to a prediction of the central film thickness development over time. This development from dry to fully flooded is presented in Fig. 8 for three different droplet frequencies.

\subsection{Experimental Validation}

The prediction of the central film thickness development over time was validated with the full setup from Fig. 1 at three different frequencies $(5 \mathrm{~Hz}, 10 \mathrm{~Hz}$, and $20 \mathrm{~Hz}$ ). The results are presented in Fig. 9. The outer region in terms of $y$ is lubricated, preliminary to the central region, until the contact remains in a fully flooded state. The film thickness was extracted from the image sequence to show its development over time at the centerline $x=0$ in Fig. 10 and the animation in Online Appendix A.4. The contact is filled with lubricant in roughly $2400 \mathrm{~s}$ using a $5 \mathrm{~Hz}$ droplet frequency, which corresponds to $40 \mathrm{~nL}$ of oil.
Table 1 Properties of the used solids/oil and test parameters

\begin{tabular}{|c|c|c|c|}
\hline & $21{ }^{\circ} \mathrm{C}$ & \multicolumn{2}{|l|}{$90{ }^{\circ} \mathrm{C}$} \\
\hline \multicolumn{4}{|c|}{ Lubricant properties: shell HVI-60 } \\
\hline$\rho_{0}$ & 900 & 855 & $\mathrm{~kg} \mathrm{~m}^{-3}$ \\
\hline$\eta_{0}$ & 46.3 & 4.4 & $\mathrm{mPa} \mathrm{s}$ \\
\hline$\alpha$ & 23.4 & - & $\mathrm{GPa}^{-1}$ \\
\hline \multirow[t]{2}{*}{$\sigma$} & 28 & 22 & $\mathrm{mN} \mathrm{m}^{-1}$ \\
\hline & Ball & Disk & \\
\hline \multicolumn{4}{|c|}{ Properties of solids } \\
\hline$R_{x}=R_{y}$ & 9.525 & $\infty$ & $\mathrm{mm}$ \\
\hline$E$ & 210 & 70 & $\mathrm{GPa}$ \\
\hline$v$ & 0.3 & 0.25 & - \\
\hline \multicolumn{4}{|c|}{ Test parameters } \\
\hline$\mu_{\mathrm{m}}$ & 300 & & $\mathrm{~mm} / \mathrm{s}$ \\
\hline$F$ & 20 & & $\mathrm{~N}$ \\
\hline$R_{\mathrm{disc}}$ & 34.5 & & $\mathrm{~mm}$ \\
\hline$L$ & 5.963 & & - \\
\hline$M$ & 170.1 & & - \\
\hline
\end{tabular}

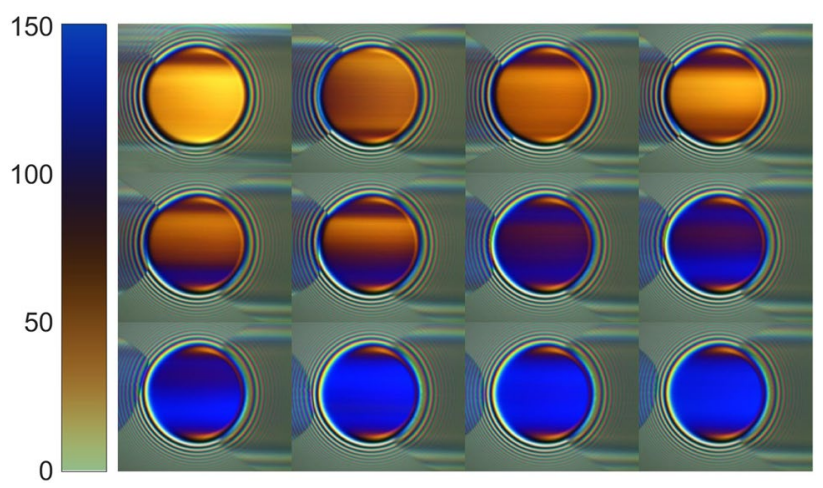

Fig. 9 Image sequence of a contact that is lubricated with a droplet supply frequency of $5 \mathrm{~Hz}$. The entrainment direction is from left to right. Time develops in lexicographic order

The central film thickness was extracted and compared to the prediction of Fig. 8, which is presented in Fig. 11 for all DOD frequencies. The central film thickness measurements are lagging compared to the prediction, since the outer region in terms of $y$ is preliminary filled. Therefore, a frequency-dependent delay of $\delta_{t}=3200 / f$ is set for the predictions. After this delay, the central film thickness develops as predicted.

The last step in the validation process was to compare all results, see Fig. 12. The experimental data are translated to dimensionless data by applying the scaling factors $H_{c}=h_{c} / c$ and $T=\left(t-\delta_{t}\right) \frac{u_{m}}{a}$. The time data for $F=4.5 \times 10^{-3}$ and $F=9.1 \times 10^{-3}$ are multiplied and quadruplicated, respectively, so all measurements can be compared to one dimensionless prediction. The film thickness follows the predicted 


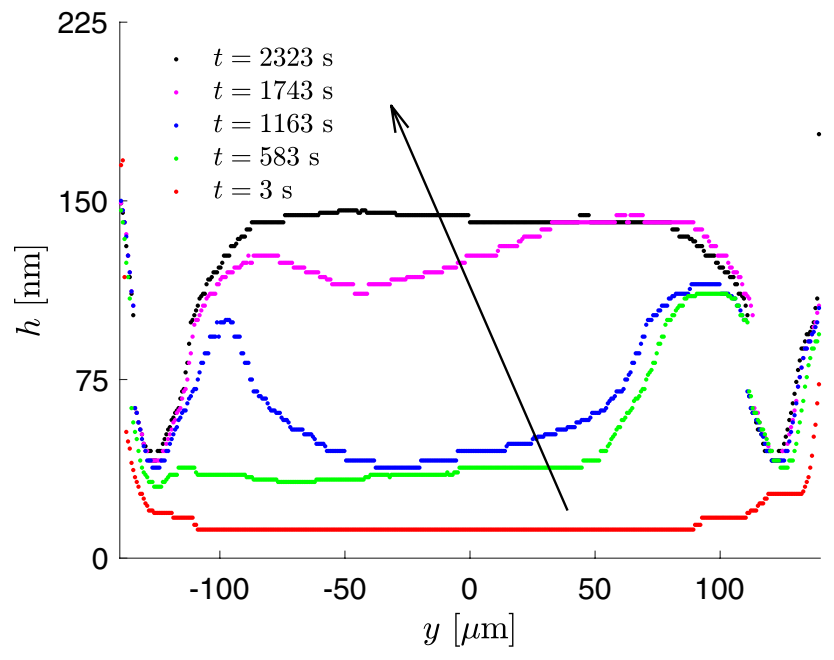

Fig. 10 Experimentally obtained film thickness at $x=0$ using a droplet supply frequency of $5 \mathrm{~Hz}$ at five different time instants. The figure shows the film thickness build-up behaviour from dry to fully flooded over $2400 \mathrm{~s}$

Table 2 Properties of the emitted HVI-60 droplet in the upward and downward directions and associated values of the relevant dimensionless numbers Weber (We) and Reynolds (Re)

\begin{tabular}{lll}
\hline & Downward & Upward \\
\hline Diameter $(\mu \mathrm{m})$ & 18.9 & 18.6 \\
Volume $(\mathrm{pL})$ & 3.5 & 3.3 \\
Velocity $\left(\mathrm{ms}^{-1}\right)$ & 0.8 & 0.9 \\
We & 5.8 & 6.7 \\
$\operatorname{Re}$ & 22 & 23 \\
\hline
\end{tabular}

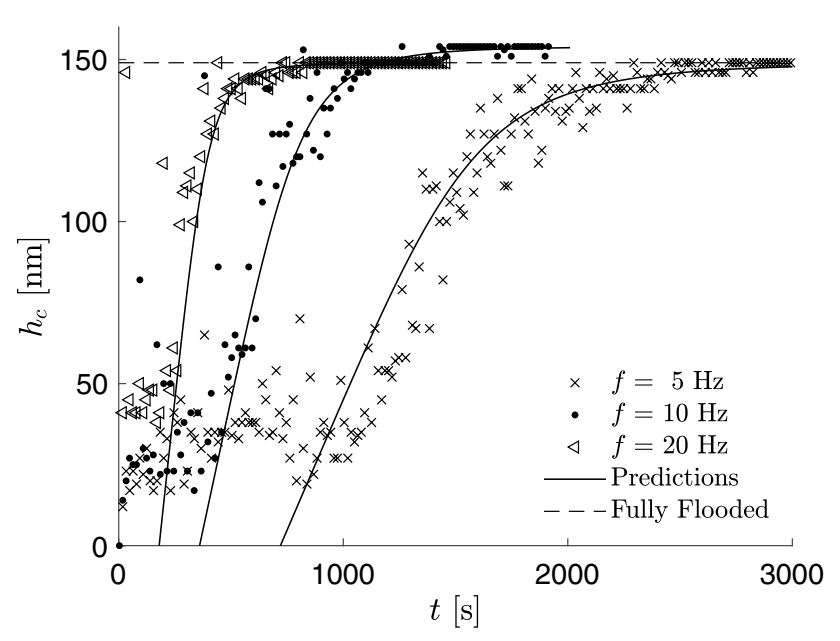

Fig. 11 Central film thickness development over time using three different droplet supply frequencies. The experiments are compared to their predictions that account for a delay of $\delta_{t}=3200 / f$ and the fully flooded film thickness trend over time for all frequencies, but the development of $F=9.1 \times 10^{-3}$ is faster and $F=2.3 \times 10^{-3}$ slower than the prediction. This is attributed to a different number of overrollings, which pushes away more oil to result in a slower film thickness development.

\section{Discussion}

In the numerical solution, the droplet is presented to the contact with its center at $Y=0$. Deviations in the position of the droplet could lead to a different lubrication process and a lower $\varepsilon$ in the replenishment model. A lower efficiency would lead to a slower central film thickness build-up in Fig. 12, and would affect $\delta_{t}$. This can be improved by a position control system to place the DOD inkjet closer to the center of the track.

In the experimental validation, the fully flooded film thickness differs from $h_{\text {cff }}=149 \mathrm{~nm}$ at $20 \mathrm{~Hz}$ and $5 \mathrm{~Hz}$ to $h_{\text {cff }} 154 \mathrm{~nm}$ at $10 \mathrm{~Hz}$ due to a difference in the room temperature of $1{ }^{\circ} \mathrm{C}$. According to the relation of Hamrock and Dowson, a viscosity increase due to $1{ }^{\circ} \mathrm{C}$ of temperature change leads to an increase of $5 \mathrm{~nm}$. This shows the sensitivity to temperature with film thickness measurements using this oil, which is also necessary to create droplets with a heated printing head.

This research is focused on single circular contacts, but the same phenomena hold for multiple elliptic and line contacts as can be seen in rolling bearings or gearboxes, respectively. By adding a shape parameter, the film build-up model can be extended to different areas of expertise. Within this

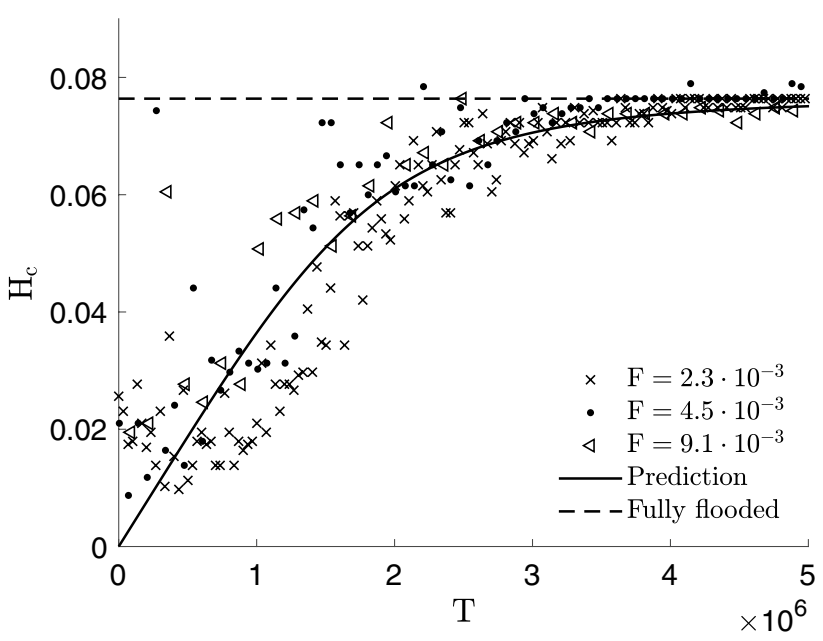

Fig. 12 Central film thickness development over time scaled to $F=2.3 \times 10^{-3}$ using three different droplet supply frequencies. The measurements are scaled to accord with dimensionless parameters and compared to the dimensionless prediction and $H_{\text {cff }}$ 
extension, the film thickness decay should be accounted for, since decay already occurs in a relative ideal setup like the one used in this research, as can be concluded from Fig. 12 . A possible solution is to use the decay model of van Zoelen [16].

\section{Conclusions}

This research shows a novel lubrication technique in EHL to maximize efficiency by minimizing the amount of oil usage, while keeping the process under full control. A single ballon-disk contact was lubricated by drop-on-demand inkjet printing using oil droplets of $3.3 \mathrm{pL}$ and $46 \mathrm{mPa}$ s. Each droplet was presented to the contact similarly in terms of size, position, and properties, which ensured control.

The single-droplet dynamics during ingestion by the contact were simulated with an accurate numerical method to show a lubrication efficiency of $85 \%$. This result was used to present a simple model to predict the film thickness over time using various droplet frequencies. The model was validated on a single ball-on-disk setup, which was lubricated from a dry contact to a fully flooded contact at three different droplet supply frequencies with a total oil usage of $40 \mathrm{~nL}$. All results are in quite good agreement with the model despite some lag in the start-up phase.

The experiments show that lubrication by drop-ondemand printing is a promising technique, which can be used with full control in an experimental setup. It can be extended to machine elements such as bearings and gears to improve control of friction by active relubrication using minute amounts of oil. This could lead to less energy losses to benefit in favor of a long-term sustainable development.

Acknowledgements The authors would like to express their thanks to Dr. M. Smeeth from the PCS Instruments for the modification of the EHL rig. Furthermore, thanks are offered to Walter Lette and Steven Wanrooij for their technical support.

Open Access This article is distributed under the terms of the Creative Commons Attribution 4.0 International License (http://creativeco mmons.org/licenses/by/4.0/), which permits unrestricted use, distribution, and reproduction in any medium, provided you give appropriate credit to the original author(s) and the source, provide a link to the Creative Commons license, and indicate if changes were made.

\section{References}

1. Holmberg, K., Erdemir, A.: Influence of tribology on global energy consumption, costs and emissions. Friction 5(3), 263-284 (2017). https://doi.org/10.1007/s40544-017-0183-5

2. Hamrock, B .J., Dowson, D.: Isothermal elastohydrodynamic lubrication of point contacts. III: fully flooded results.
ASME J. Lubr. Technol. 99(2), 264-275 (1977). https://doi. org/10.1115/1.3453074

3. Nijenbanning, G., Venner, C.H., Moes, H.: Film thickness in elastohydrodynamically lubricated elliptic contacts. Wear 176(2), 217-229 (1994). https://doi.org/10.1016/0043-1648(94)90150-3

4. Wedeven, L.D., Evans, D., Cameron, A.: Optical analysis of ball bearing starvation. ASME J. Lubr. Technol. 93, 349-363 (1971). https://doi.org/10.1115/1.3451591

5. Booser, E.R., Wilcock, D.F.: Minimum oil requirements of ball bearings. Lubr. Eng. 9, 140-161 (1953)

6. Pemberton, J., Cameron, A.: A mechanism of fluid replenishment in elastohydrodynamic contacts. Wear 37, 185-190 (1976). https ://doi.org/10.1016/0043-1648

7. Chevalier, F.: Modélisation des conditions d'alimentation dans les contacts élastohydrodynamiques ponctuels. Ph.D. thesis, INSA, 1996ISAL0124 (1996)

8. Chevalier, F., Lubrecht, A .A., Cann, P .M .E., Colin, F., Dalmaz, G.: Starvation phenomena in E.H.L. point contacts: influence of inlet flow distribution. Tribol. Ser. 31, 213-223 (1996). https:// doi.org/10.1016/S0167-8922(08)70785-5

9. Chevalier, F., Lubrecht, A.A., Cann, P.M.E., Colin, F., Dalmaz, G.: Film thickness in starved EHL point contacts. J. Tribol. 120, 126-133 (1998). https://doi.org/10.1115/1.2834175

10. Damiens, B.: Modélisation de la lubrification sous-alimentée dans les contacts élastohydrodynamiques elliptiques. Ph.D. thesis, L'INSA de Lyon (2003)

11. Damiens, B., Venner, C.H., Cann, P.M.E., Lubrecht, A.A.: Starved lubrication of elliptical EHD contacts. ASME. J. Lubr. Technol. 126(1), 105-111 (2004). https://doi.org/10.1115/1.1631020

12. Wijnant, Y.H., Venner, C.H.: Contact dynamics in starved elastohydrodynamic lubrication. Elsevier Tribol. Ser. 36, 705-716 (1999). https://doi.org/10.1016/S0167-8922(99)80089-3

13. Venner, C.H., Berger, G., Lugt, P.M.: Waviness deformation in starved EHL circular contacts. ASME J. Lubr. Technol. 126, 248-257 (2003). https://doi.org/10.1115/1.1572514

14. Venner, C .H., Popovici, G., Lugt, P .M., Organisciak, M.: Film thickness modulations in starved elastohydrodynamically lubricated contacts induced by time-varying lubricant supply. ASME J. Lubr. Technol. 130(4), 041501-041501-10 (2008). https://doi. org/10.1115/1.2958069

15. van Zoelen, M.T., Venner, C.H., Lugt, P.M.: Prediction of film thickness decay in starved elasto-hydrodynamically lubricated contacts using a thin layer flow model. Proc. Inst. Mech. Eng. Part J 223(3), 541-552 (2009). https://doi.org/10.1243/13506 501JET524

16. van Zoelen, M.T., Venner, C.H., Lugt, P.M.: The prediction of contact pressure-induced film thickness decay in starved lubricated rolling bearings. Tribol. Trans. 53(6), 831-841 (2010). https ://doi.org/10.1080/10402004.2010.492925

17. van Zoelen, M.T.: Thin layer flow in rolling element bearings. Ph.D. thesis, University of Twente, Netherlands (12 2009). https ://doi.org/10.3990/1.9789036529341

18. Le, H.P.: Progress and trends in ink-jet printing technology. J. Imaging Sci. Technol. 42, 49-62 (1999)

19. Zoltan, S.L.: (Clevite Corp.), Pulse droplet ejection system. U.S. Patent 3683212

20. Vaught, J.L., Cloutier, D.K., Donald, K., Meyer, J.D., Tacklind, C.A., Taub, H.H.: Thermal ink jet printer. U.S. Patent 4490728

21. van der Meulen, M.P.: Meniscus motion and drop formation in inkjet printing. Ph.D. thesis, University of Twente (2 2015). https ://doi.org/10.3990/1.9789036538282

22. Staat, H.J.J.: Droplet dynamics during flight, impact and evaporation. Ph.D. thesis, University of Twente. (2016). https://doi. org/10.3990/1.9789036540810 
23. Fraters, A.B.: Inkjet printing: bubble entrainment and satellite formation. Ph.D. thesis, University of Twente, Netherlands. (2018). https://doi.org/10.3990/1.9789036546645

24. Li, X.M., Guo, F., Wang, S., Liu, C.L., Wang, W.: Behaviors of a micro oil droplet in an EHL contact. Friction 4(4), 359-368 (2016)

25. Liu, C.L., Guo, F., Li, X.M., Li, S.Y., Han, S.L., Wan, Y.: Experimental study of elastohydrodynamic lubrication behaviour under single oil droplet supply. Tribol. Int. 118, 432-440 (2017). https ://doi.org/10.1016/j.triboint.2017.10.020

26. Li, X.M., Guo, F., Wong, P.L., Zhao, Y.: Regulation of lubricant supply by wettability gradient in rolling EHL contacts. Tribol. Int. 120, 565-574 (2018). https://doi.org/10.1016/j.tribo int.2018.01.020

27. Li, S.Y., Guo, F., Li, X.M., Liu, C.L.: Numerical simulation of an oil droplet passing through an EHL contact. Tribol. Int. 112(3), 58-67 (2017). https://doi.org/10.1016/j.triboint.2017.03.033

28. Vegte, S., Venner, C.: Inkjet based droplet on demand lubrication system for ehl. In: Proceedings of the WTC 2017, World Tribology Congress 2017 Beijing (2017)

29. Elrod, H.G.: A cavitation algorithm. ASME J. Lubr. Technol. 103(3), 350-354 (1981). https://doi.org/10.1115/1.3251669

30. Dowson, D., Higginson, G.R.: Elasto-Hydrodynamic Lubrication, International Series on Materials Science and Technology. Pergamon Press, Oxford (1977)

31. Roelands, C.: Correlational aspects of the viscosity-temperaturepressure relationship of lubricating oils. Ph.D. thesis, TU Delft (1966)

32. Venner, C.H., Morales-Espejel, G.E.: Amplitude reduction of small-amplitude waviness in transient elastohydrodynamically lubricated line contacts. Proc. Inst. Mech. Eng. Part J 213(6), 487-504 (1999). https://doi.org/10.1243/1350650991542848

33. Wijnant, Y.H.: Contact dynamics in the field of elastohydrodynamic lubrication. Ph.D. thesis, Universiteit Twente (1998)

34. Venner, C.H., Lubrecht, A.A.: Multi-level Methods in Lubrication, vol. 37. Elsevier, Amsterdam (2000)

35. Westlake, F.J., Cameron, A.: Fluid film interferometry in lubrication studies. Nature 214, 633-634 (1967). https://doi. org/10.1038/214633a0
36. Foord, C.A., Wedeven, L.D., Westlake, F.J., Cameron, A.: Optical elastohydrodynamics. Proc. Inst. Mech. Eng. 184(1), 487-505 (1969)

37. Johnston, G.J., Wayte, R., Spikes, H.A.: The measurement and study of very thin lubricant films in concentrated contacts. Tribol. Trans. 34(2), 187-194 (1991). https://doi.org/10.1080/10402 009108982026

38. Cann, P.M.E., Spikes, H.A., Hutchinson, J.: The development of a spacer layer imaging method (SLIM) for mapping elastohydrodynamic contacts. Tribol. Trans. 39(4), 915-921 (1996). https:// doi.org/10.1080/10402009608983612

39. Chen, A.U., Basaran, O.A.: A new method for significantly reducing drop radius without reducing nozzle radius in drop-on-demand drop production. Phys. Fluids 14(1), L1-L4 (2002). https://doi. org/10.1063/1.1427441

40. Duineveld, P., Dijksman, J.: Ultra small droplet generation in inkjet printing by higher order meniscus oscillations. In: Proceedings of the NIP \& Digital Fabrication Conference, pp. 147-150 (2018). https://doi.org/10.2352/ISSN.2169-4451.2018.34.147

41. Bos, A., Zijlstra, A., Gelderblom, E., Versluis, M.: iLIF: illumination by laser-induced fluorescence for single flash imaging on a nanoseconds timescale. Exp. Fluids 51, 1283-1289 (2011). https ://doi.org/10.1007/s00348-011-1146-7

42. Stow, C .D., Hadfield, M .G.: An experimental investigation of fluid flow resulting from the impact of a water drop with an unyielding dry surface. Proc. R. Soc. Lond. A 373, 419-441 (1981). https://doi.org/10.1098/rspa.1981.0002

43. Reis, N., Derby, B.: Ink jet deposition of ceramic suspensions: modeling and experiments of droplet formation. MRS Proc. 625, 117-122 (2000). https://doi.org/10.1557/PROC-625-117

44. Duineveld, P.C., de Kok, M.M., Buechel, M., Sempel, A., Mutsaers, K.A.H., van de Weijer, P., Camps, I.G.J., van de Biggelaar, T., Rubingh, J.J.M., Haskal, E.I.: Ink-jet printing of polymer light-emitting devices. Proc. SPIE 4464, 59-67 (2002). https:// doi.org/10.1117/12.457460 